\title{
Molecular Origin of Contact Line Friction in Dynamic Wetting
}

\author{
Petter Johansson and Berk Hest* \\ Department of Physics and Swedish e-Science Research Center, \\ KTH Royal Institute of Technology, Stockholm, Sweden
}

(Dated: February 20, 2018)

\begin{abstract}
A hydrophilic liquid, such as water, forms hydrogen bonds with a hydrophilic substrate. The strength and locality of the hydrogen bonding interactions prohibit slip of the liquid over the substrate. The question then arises how the contact line can advance during wetting. Using large-scale molecular dynamics simulations we show that the contact line advances by single molecules moving ahead of the contact line through two distinct processes: either moving over or displacing other liquid molecules. In both processes friction occurs at the molecular scale. We measure the energy dissipation at the contact line and show that it is of the same magnitude as the dissipation in the bulk of a droplet. The friction increases significantly as the contact angle decreases, which suggests suggests thermal activation plays a role. We provide a simple model that is consistent with the observations.
\end{abstract}

\section{INTRODUCTION}

The dynamics of wetting has been studied for more than a century. The advent of new technological applications combined with advances in both experimental and simulation techniques has led to increased attention in the last decades [1] 3 .

Droplet spreading on a substrate is commonly characterized by two stages: an early stage where inertial forces oppose droplet spreading [4, 6] followed by a later one where viscous forces dominate [7, 8]. Hydrodynamics along with a balance of surface tensions explains most details of the wetting process. But there are still aspects which are not well understood. In particular it is unclear what processes govern the dynamics of the three-phase contact line between liquid, substrate and vapor shown in figure 1 While hydrodynamical models can incorporate these processes as boundary conditions they give no hint as to what they are. A detailed, molecular picture is needed to understand what is happening in the contact line region.

Two different situations can be distinguished in dynamic wetting. The simpler case is a substrate-liquid combination with nanoscale slip. Here there are no incompatibilities with hydrodynamics 9]. The more complicated case occurs with the no-slip boundary condition, as is common when both liquid and substrate are hydrophilic. In a continuum description, no slip is fundamentally incompatible with contact line motion. Here we will focus on the no-slip case.

It has been shown that hydrodynamical models can reproduce experimental data by accounting for the energy dissipation inside the bulk and at the contact line [10]. The nature of this dissipation at the contact line is unknown. Perrin et al. relate it to the contact line pinning to nanoscopic defects present at the substrate [11] but molecular simulation studies have shown that it also appears on completely smooth, defect-free substrates. In particular Nakamura et al. show that the dissipation in their system can be explained by liquid slip against the substrate [9], but it has also been seen in similar smooth systems where the liquid cannot slip over the substrate [12. This suggests that there is an additional contribution to contact line dissipation due purely to how the contact line advances on a molecular level: a molecular contact line friction [13, 14. While it is likely that several or all of these modes of energy dissipation are present in macroscopic wetting it is important to know where and how they contribute.

A recent study has shown that the no-slip condition in dynamic wetting is a consequence of liquid molecules forming hydrogen bonds with substrate molecules [12. Hydrogen bonds are a particular class of transient and non-covalent electrostatic bonds between polar molecules, such as water. They are characterized by being significantly stronger and more directional than van der Waals interactions, but weaker than covalent bonds. Because the strength of a hydrogen bond is an order of magnitude larger than the thermal energy,
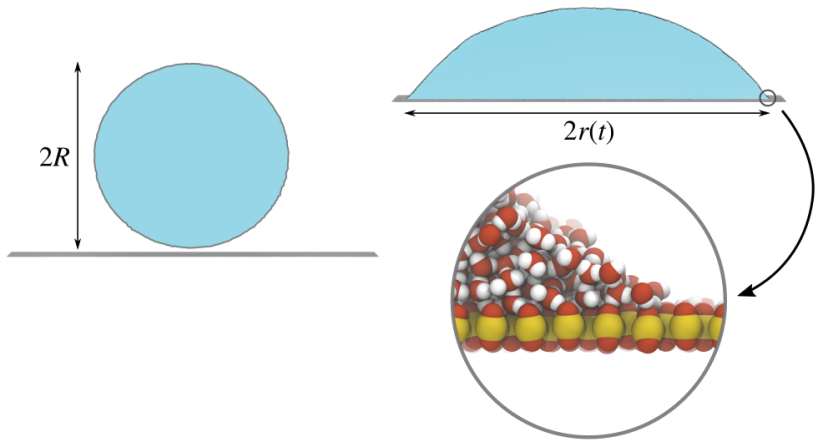

Figure 1: The simulated systems are droplet cylinders with $R=50 \mathrm{~nm}$, consisting of 1.2 million water molecules. After making contact with the hydrophilic substrates they spread out with a base radius $r(t)$.

Zooming in shows the molecular nature of the droplets, particularly important at the contact line. 


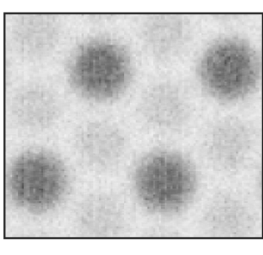

(a)
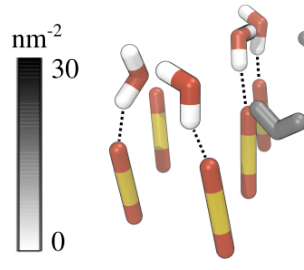

(b)
Figure 2: a) A sampled 2D number density plot of hydrogen atoms in the first layer of water molecules shows that they are localized (hydrogen bonded) to the underlying $\mathrm{SiO}_{2}$ "molecules." b) A snapshot of hydrogen bonded molecules. All but the water molecules in gray are hydrogen bonded.

these "bonds" localize the liquid molecules to the underlying substrate molecules, thereby preventing slip. Most atomistic studies of wetting have been performed with simple Lennard-Jones type models for the substrate and often for the liquid as well [15 18]. Simulations with models that capture hydrogen bonding have been limited to smaller droplets [19] or studied the effects of hydrogen bonds within the liquid itself, not between liquid and substrate [20, 21]. We will show that the dynamics of water spreading on a hydrogen-bonding substrate is qualitatively different from Lennard-Jones models, in particular at the contact line.

In this paper we use atomistic molecular dynamics (MD) simulations to measure molecular contact line friction for a semi-realistic quasi-2D system of water on silica with several hydrophilic static contact angles. The setup is detailed in figure 1. Our system is large in order to capture slow, long-term behavior and to provide sufficient statistics. Using complex interactions between the substrate and water we replicate replicate realistic system set-ups and in particular the no-slip boundary condition through the formation of liquid-substrate hydrogen bonds. We observe that in no-slip wetting the contact line friction is related to the dynamics of the contact line. We propose a molecular explanation for this dissipation and show that it matches our results.

\section{SYSTEM SET-UP}

We use an idealized silica substrate to study hydrophilic wetting. It is constructed as a monolayer of $\mathrm{SiO}_{2}$ "molecules" consisting of two negatively charged oxygen atoms and a positively charged silica atom in the middle. These molecules do not have net charge nor a dipole moment, so there is no long-range interaction with water. They are set in a triangular formation with a spacing of $0.45 \mathrm{~nm}$.

The polar nature of the substrate molecules give rise to

\begin{tabular}{lccccc}
\hline \hline Substrate & $q_{\mathrm{O}}(e)$ & $\lambda(\mathrm{nm})$ & $\theta_{0}\left(^{\circ}\right)$ & $\left(\gamma_{S V}-\gamma_{S L}\right) / \gamma$ & $\hat{\mu}_{f}(\mu)$ \\
\hline Silica & -0.84 & $0.0 \pm 0.1$ & 0 & 1.9 & 110 \\
Silica & -0.79 & $0.0 \pm 0.1$ & 0 & 1.3 & 94 \\
Silica & -0.74 & $0.0 \pm 0.1$ & $36 \pm 0.2$ & $\cos \theta_{0}=0.81$ & 76 \\
Silica & -0.67 & $0.0 \pm 0.1$ & $70 \pm 0.3$ & $\cos \theta_{0}=0.34$ & 57 \\
Atom. LJ & - & $1.1 \pm 0.1$ & $37 \pm 0.4$ & $\cos \theta_{0}=0.80$ & - \\
\hline \hline
\end{tabular}

Table I: Characteristics of the studied substrates. The atoms of the silica molecules $\left(\mathrm{SiO}_{2}\right)$ carry charges according to $q_{\mathrm{Si}}=-2 q_{\mathrm{O}}$ where the oxygen charge $q_{\mathrm{O}}$ is listed in the table, $e$ is the elementary charge. The atomistic LJ substrate atoms are non-charged which results in water slipping across it. $\hat{\mu}_{f}$ is a

substrate-dependent friction coefficient factor measured and described later for the no-slip substrates.

strong, transient hydrogen bonds with water molecules. The strength of these short-ranged bonds result in a single, layer of non-slipping water molecules "bound" to the substrate molecules. Individual water molecules exchange between this layer and the bulk with an average time of $24 \mathrm{ps}$ for our systems. This creates the no-slip condition between the substrate and the liquid, as the bottom layer of molecules cannot move freely (figure 21.

The wetting energetics of partially wetting systems is related to the static contact angle $\theta_{0}$ through Young's equation as $\gamma \cos \theta_{0}=\gamma_{S V}-\gamma_{S L}$ where $\gamma \equiv \gamma_{L V}, \gamma_{S V}$ and $\gamma_{S L}$ respectively are the liquid-vapor, solid-vapor and solid-liquid surface tensions. For perfectly wetting systems $\gamma_{S V}-\gamma_{S L} \geq \gamma \cos \theta_{0}$. By tuning the atomistic charges of our silica molecules we change the substrateliquid interactions to construct no-slip substrates with static contact angles ranging from $0^{\circ}$ to $70^{\circ}$. To assert that the water does not slip across the substrate, we use a Couette flow set-up. By measuring the flow gradient we calculate that the slip length $\lambda$ for all of our hydrophilic silica systems is $0.0 \pm 0.1 \mathrm{~nm}$.

We compare the results to a simpler system of water on a hydrophobic substrate of Lennard-Jones atoms (see [12). Since water cannot hydrogen bond to this substrate, slip occurs. We measure a slip length of $1.1 \pm 0.1 \mathrm{~nm}$ in an identical Couette flow set-up. Details of the characteristics of all substrates are provided in table 1 .

The water droplets are modeled as cylinders with an initial radius $R$ of $50 \mathrm{~nm}$ and periodic width $w$ of 4.67 $\mathrm{nm}$. They contain a total of 1.2 million water molecules, or 3.6 million atoms, each. We chose SPC/E [22] as the water model, which is characterized by its surface tension $\gamma=5.78 \cdot 10^{-2} \mathrm{Pam}$, viscosity $\mu=8.77 \cdot 10^{-4} \mathrm{Pas}$ and density $\rho=986 \mathrm{~kg} \mathrm{~m}^{-3}$. With the capillary speed $v_{\mathrm{c}} \equiv \gamma / \mu$ the Reynold's number of the system is $\operatorname{Re} \equiv$ $\rho v_{\mathrm{c}} R / \mu=3.7$. See the appendix for details on the simulation procedure. 

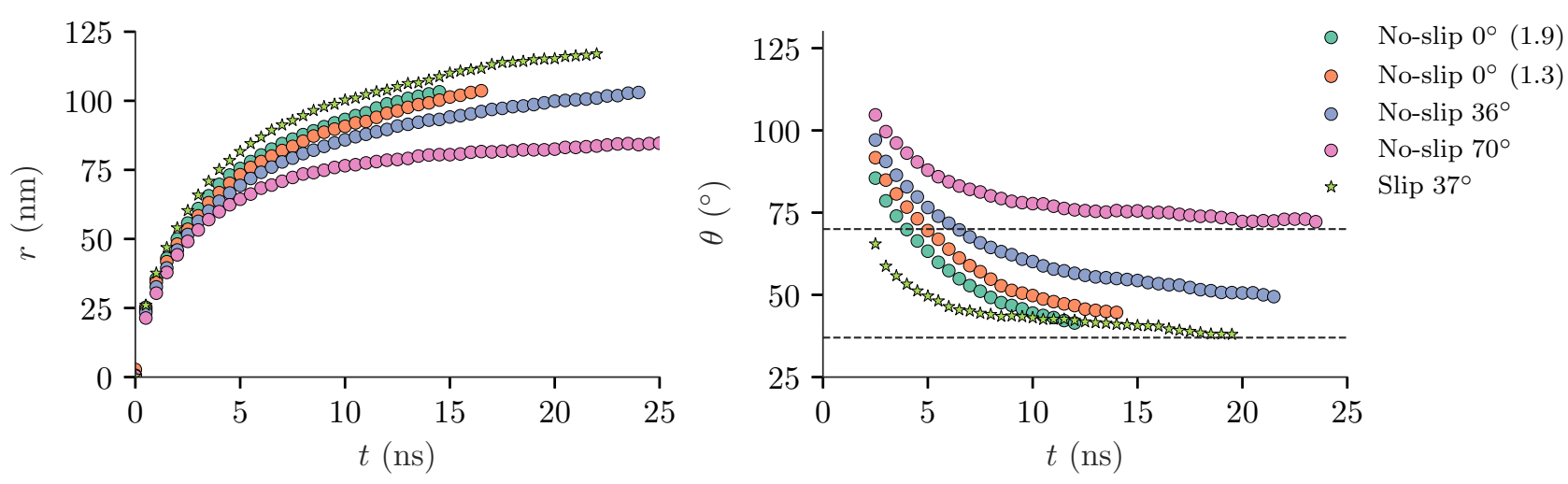

Figure 3: Measurements of the wetting radius $r(t)$ (left) and the dynamic contact angle $\theta(t)$ (right) for simulations on all involved substrates. The dashed lines denote the two equilibrium states of angles $36^{\circ}$ and $70^{\circ}$.

\section{SIMULATION RESULTS}

To study the dynamics of our wetting systems we sample our system in two-dimensional bins during the simulation. Droplet interfaces are identified using a simple density cutoff. Note that apart from the wetting radius $r$ (figure 1) all measurements presented here have been center averaged over $5 \mathrm{~ns}$ of data to decrease the influence of thermal noise.

At each time $t$ the wetting radius $r(t)$ is taken as half of the droplet base extension and the dynamic contact angle $\theta(t)$ is measured at a height of $1 \mathrm{~nm}$ above the substrate. These are shown in figure 3 . We see that the addition of slip allows for much faster relaxation towards the equilibrium compared to the no-slip systems, even compared to the perfectly wetting system. The no-slip systems have very similar dynamics in the early inertial regime, diverging only as they transition into the viscous regime. Over the simulations the no-slip substrate with a contact angle of $70^{\circ}$ and the substrate with slip get close to their equilibrium states (dashed lines) and their dynamics slow down dramatically.

Ideally one would like to measure this dissipation directly from the contact line-local shear stress. However, this is in practice unfeasible using molecular simulations since the thermal velocity of molecules is roughly 100 times higher than the flow velocity (see Supplementary Material at [URL] for an extracted shear field which has been averaged over 200 frames separated by 5 ps). Thus we resort to modeling. When considering the modes of energy dissipation during dynamic wetting the bulk and interfacial dissipation terms are well characterized by continuum models. The contact line dissipation rate is given by [13, 23 .

$$
\dot{E}_{\mu_{f}} \sim 2 w \mu_{f} v^{2}
$$

where $v=d r / d t$ is the contact line velocity, $2 w$ is the total length of the two contact lines and $\mu_{f}$ is referred to as a contact line friction coefficient which has units

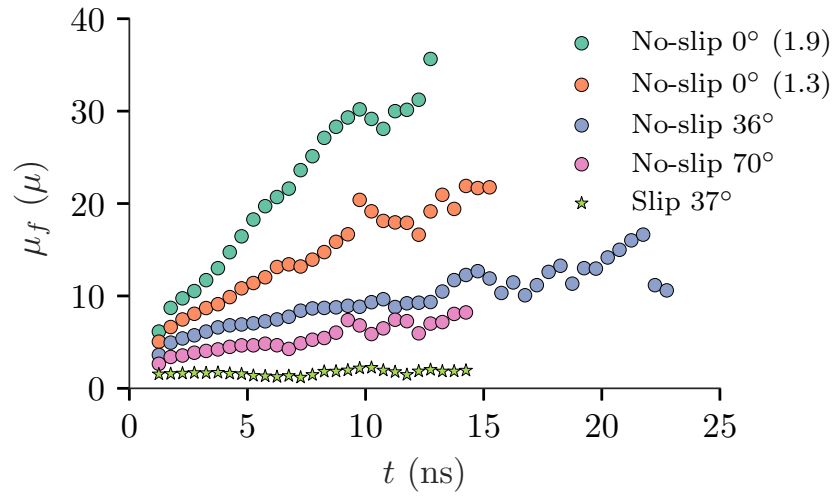

Figure 4: The measured contact line friction coefficient $\mu_{f}$ in units of viscosity $\mu$ for all substrates. When the liquid cannot slip the coefficient increases as the droplet wets the substrate.

of viscosity. Furthermore, in a damped regime the contact line velocity is related to the force $F_{\mathrm{cl}}$ acting on the contact line through the friction coefficient (see 24] for a derivation and discussion in the context of the phase-field model):

$$
v=\frac{F_{\mathrm{cl}}}{\mu_{f}}=\frac{\gamma_{S V}-\gamma_{S L}-\gamma \cos \theta}{\mu_{f}} .
$$

This result is a direct relation between the contact line friction factor $\mu_{f}$ and the two dynamic measurables $v(t)$ and $\theta(t)$, given the system energetics. This allows us to measure the contribution of the contact line friction to our dynamics.

Figure 4 shows the contact line friction coefficient $\mu_{f}$ calculated for each time and system using (2). There are a few things of note here: The first and most obvious feature is that although $\mu_{f}$ has been thought of as a constant which varies only between different substrates, we see that it increases during the wetting process for our no-slip systems. Second is that the system where slip is present has an apparent constant, non-zero friction co- 


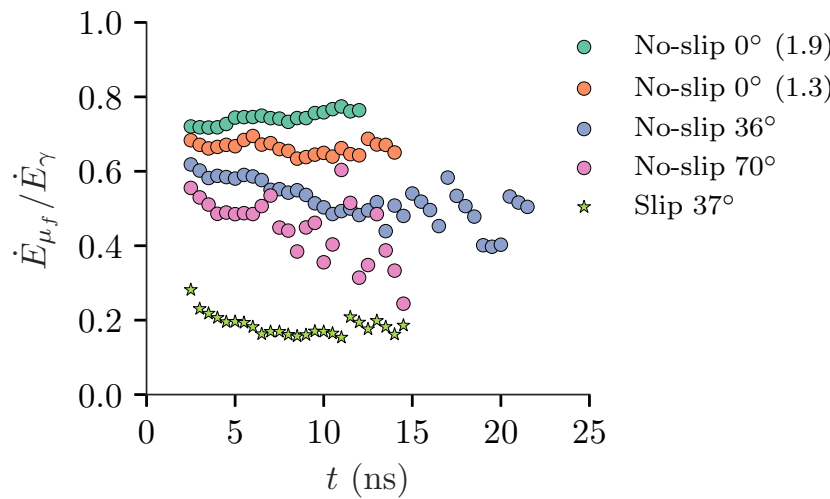

Figure 5: Contact line dissipation rate $\dot{E}_{\mu_{f}}$ as a fraction of the energy gained as the droplet wets the substrate $\dot{E}_{\gamma}$. For the no-slip substrates around half of the gained energy is dissipated at the contact line.

efficient during its entire relaxation. Note that the data for the silica with $\theta_{0}=70^{\circ}$ and the slip substrate is cut at $15 \mathrm{~ns}$. This is due to the systems being very close to equilibrium (figure 3, making the measurement of $v$ (and thus $\mu_{f}$ ) dominated by thermal fluctuations.

To see whether or not the contact line friction is important to consider we use (1) to calculate the dissipation rate and compare it to the net energy $\dot{E}_{\gamma}$ being fed into the system as it wets the substrate

$$
\dot{E}_{\gamma}=w\left[2 v\left(\gamma_{S V}-\gamma_{S L}\right)-\gamma \dot{s}\right]
$$

where $\dot{s}(t)$ is the rate at which the length of the liquidvapor interface changes, which we measure from our simulations, and $w$ as before is the interface (cylinder) width. A comparison between $\dot{E}_{\mu_{f}}$ and $\dot{E}_{\gamma}$ is shown in figure 5 . For the no-slip substrates the dissipation at contact line accounts for more than half of the energy gained as the droplet wets. Even for the substrate with slip, a quarter of the gained energy is dissipated at the contact line. As such, contact line friction is an important feature to consider when modeling similar systems. Note that the measurement error goes up heavily as $v$ and $\dot{s}$ approach zero, which leads to a large variance for the no-slip system with $70^{\circ}$ static contact angle, since it quickly approaches its equilibrium state.

\section{ENERGY DISSIPATION IN CONTACT LINE FRICTION}

Having shown that the contact line friction increases during the simulations of our no-slip systems, we are naturally invited to consider why that is. This question ties into the mechanism(s) of contact line advancement. For the case of a no-slip system, we identify two important modes, sketched in figure 6. The first mode is a molecule from the non-bonded layers of liquid molecules rolling

\begin{tabular}{cccccc}
\hline \hline$t(\mathrm{~ns})$ & $r(\mathrm{~nm})$ & $v(\mathrm{~m} / \mathrm{s})$ & $\theta$ & $\mu_{f}(\mu)$ & $f_{\mathrm{MKT}}$ \\
\hline 2.5 & 50 & 14 & $95^{\circ}$ & $4.4 \pm 0.5$ & $0.19 \pm 0.01$ \\
8.0 & 80 & 3.0 & $64^{\circ}$ & $9 \pm 1$ & $0.49 \pm 0.01$ \\
12.5 & 90 & 1.7 & $55^{\circ}$ & $12 \pm 2$ & $0.49 \pm 0.01$ \\
\hline
\end{tabular}

Table II: Data collected from simulations on the no-slip $36^{\circ}$ silica just in the switch from the inertial to viscous wetting (at $2.5 \mathrm{~ns}$ ) and at two stages of the viscous regime (at $8.0 \mathrm{~ns}$ and $12.5 \mathrm{~ns}$ ). The contact line speed $v$ decreases by an order of magnitude and the fraction $f_{\text {MKT }}$ of molecules which advanced the contact line through MKT-like jumps approaches 0.5 in the viscous regime.

onto the substrate from above (figure 6a). The second a molecule at the contact line breaking its bond to the substrate and jumping to an adjacent potential well of the substrate (figure 6b). This is the base of molecular kinetic theory (MKT) 25].

We measure which of these modes dominate during wetting using our molecular trajectories. This is done by extracting the simulation state at points in the simulation and replicating the system four times along the cylinder axis to obtain more data and better statistics. We extract states from the no-slip silica system with $\theta_{0}=36^{\circ}$ at three points: the first from when the system transitions from the inertial regime to the viscous and the second two at states within the viscous regime, when jumps between adjacent sites will be more prominent. Data is collected over a period of 1 ns with a spacing of 1 ps. When a contact line advancement event is detected, the molecule which advanced is back-traced in time. The fraction $f_{\mathrm{MKT}}$ of which arrive from an adjacent lattice site (corresponding to (b) in figure 60 is calculated from the results and reported in table II] In the viscous regime the two described modes contribute about equally to contact line advancement.

Let us consider the rolling mode (a) in more detail. Molecule $\mathrm{A}$ in figure 6a cannot drop down to the substrate without passing molecule B, which acts as an obstacle. To pass this obstacle the molecule has to move into a position from which it can drop down. The average thermal velocity of a water molecule of $370 \mathrm{~m} / \mathrm{s}$ is two orders of magnitude larger than the local flow velocity $(0.5-5 \mathrm{~m} / \mathrm{s}$ in the viscous regime). Thermal fluctuations dominate over an average mass flow in the vicinity of the contact line. Thus molecule A will not simply roll into position to drop down: it has to cross an energy barrier $\Delta E$. This barrier is due to the molecule not wanting to break bonds with neighboring molecules. It has to cross this by gaining sufficient momentum through a thermal fluctuation. After crossing the barrier, the molecule will be pulled towards the substrate and form a hydrogen bond with it. 


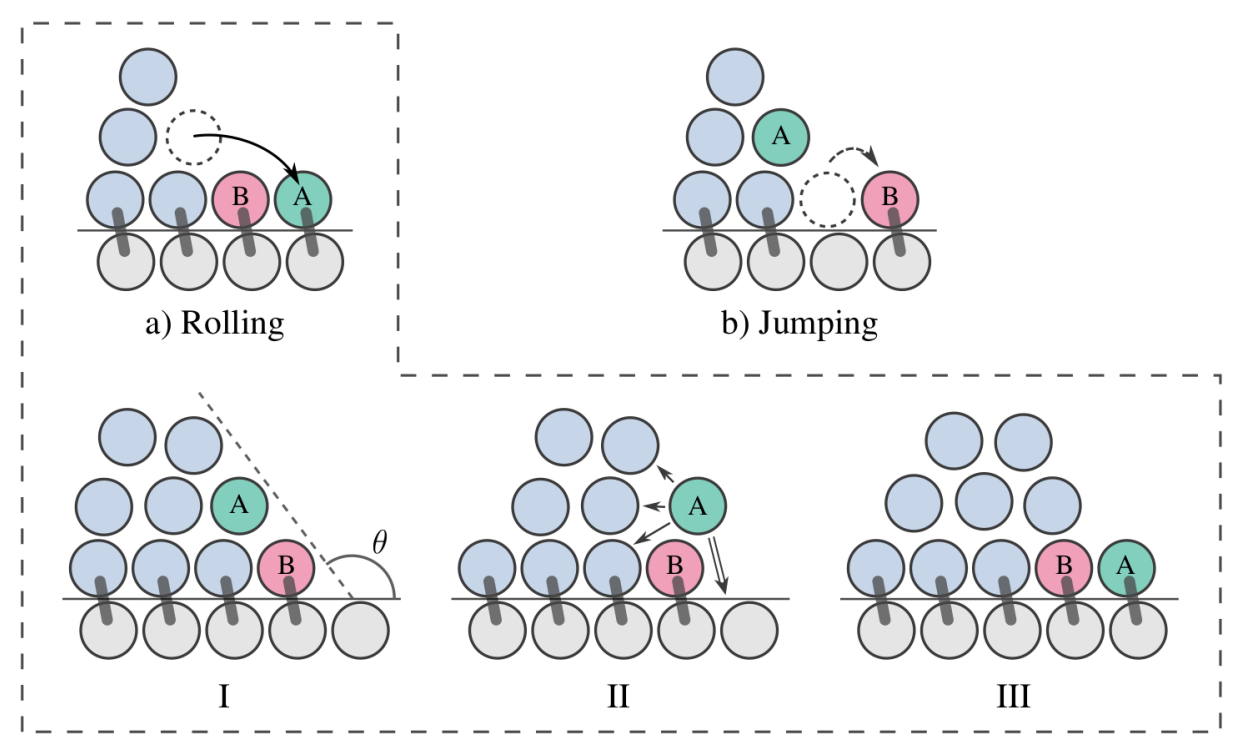

Figure 6: Modes of contact line advancement for a no-slip system. The bottom layer of liquid molecules are hydrogen bonded to the substrate. a) A molecule A rolls in from an upper layer to advance the contact line. To advance from (I) it has to pass the intermediate state (II). Its interactions with neighboring molecules lead to an energy barrier that has to be crossed through a thermal fluctuation of the interface. After crossing it, it is pulled towards the substrate (III). b) The outermost molecule B jumps to a neighboring lattice site. Described by molecular kinetic theory.

At short range the interactions of atoms in the water molecule with the substrate increase with Coulomb's law as $1 / r$. Thus most of the gain in kinetic energy occurs at the last moment, while the molecule is moving close to vertically. As the molecule hydrogen bonds to the substrate, it suddenly stops. This means that a large part of the vertical acceleration is not transferred to subsequent water molecules. Rather those molecules bump into the molecules that just advanced the contact line and mostly bounce back. Thus part of the gain in surface energy is transformed into random thermal motion in the contact line region. This heat will slowly dissipate into the environment. This is how contact line friction arises when a hydrophilic liquid spreads on a smooth, hydrophilic substrate.

We will now describe a simple model that explains why the contact line friction coefficient increases with decreasing contact angle. For the outermost molecule in the second layer to reach the transition state, it needs to horizontally travel, on average, a distance of $\Delta x=\tan \left(90^{\circ}-\theta\right)+c$ water molecule diameters. In state (II) in figure 6 the transition state is drawn as the water molecule extending by half its diameter beyond the molecule at the contact line. We take this state as the transition state and thus set $c=0.5$ (note that we cannot measure $c$ accurately). The energy required to create such a fluctuation is given by the increase in liquid-vapor interface area. This increase is proportional the square of the displacement: $\Delta E \propto(\sin \theta \Delta x)^{2}$, where the sine

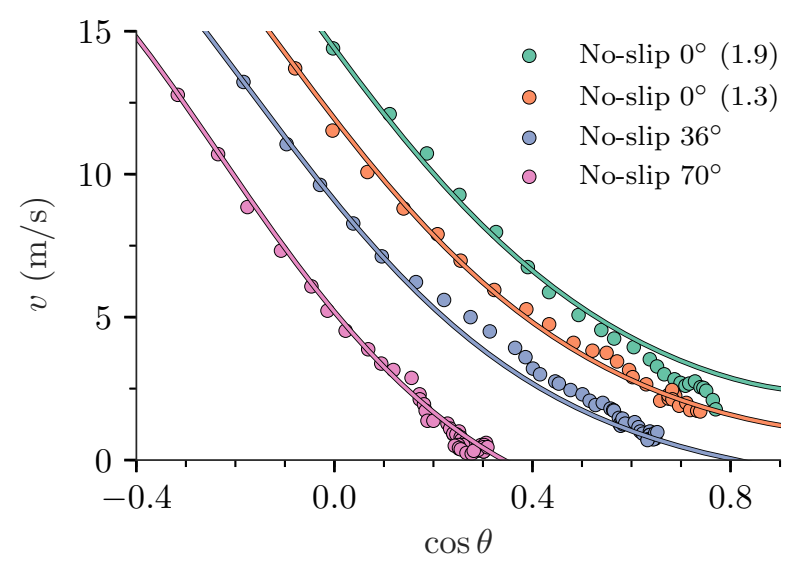

Figure 7: The contact line friction modeled as a geometry dependent energy barrier through the contact angle $\theta$. A good agreement is reached between such a model (lines) and the measured velocities (circles) in late-stage wetting for all bar the extremely hydrophilic system.

accounts for the difference in direction of $\Delta x$ and the normal of the liquid-vapor interface. For a thermally activated process, the rate decreases exponentially with the activation energy and we obtain (compare to $(2)$ ):

$$
v=\frac{e^{-\Delta E}}{\hat{\mu}_{f}} F_{\mathrm{cl}}=\frac{e^{-a\left(\sin \theta\left[\tan \left(90^{\circ}-\theta\right)+0.5\right]\right)^{2}}}{\hat{\mu}_{f}} F_{\mathrm{cl}}
$$


where $\hat{\mu}_{f}$ and $a$ are free model parameters. We cannot fit all data using a single value for $\hat{\mu}_{f}$. It is optimized for each substrate. The best fit for this model is presented in figure 7 for our non-slip substrates with the values for $\hat{\mu}_{f}$ reported per-system in tableI Iand the value $a=1.11$. We see that it provides a good prediction of the friction for most systems. Only the most attractive substrate with $\left(\gamma_{S V}-\gamma_{S L}\right) / \gamma=1.9$ shows a deviation larger than the statistical accuracy in the late stage. The good fit suggests that the observed time dependence of the friction can be explained by a dependence on the local geometry of the contact line.

Returning to the MKT-like mode, we can see how this fits into the model. The MKT jump itself involves an energy barrier, but a forward fluctuation of the second layer is also required to fill the vacancy in the first layer. The amplitude of this fluctuation is about one water molecule diameter less than the fluctuation for the rolling mode. Since the activation energy of the MKT jump is approximately constant and the activation energy of the second layer is proportional to the square of the distance, the rolling mode will dominate at large contact angles, while the MKT mode will increasingly dominate as the angle decreases. This is exactly what we observe (see table II).

Finally we consider the substrate dependence of $\hat{\mu}_{f}$. In the early stages of wetting, when $\theta$ is larger than $90^{\circ}$, the energy barrier is negligible and the friction is dominated by $\hat{\mu}_{f}$. Here water molecules can be pulled directly to the substrate. As described before, a significant part of the attraction occurs very close to the substrate and most of that energy gain is lost in collisions. The attraction between water and substrate, as given by $\gamma_{S V}-\gamma_{S L}$, increases much faster than the charge difference. Thus most of the difference in acceleration of water molecules to the different substrates occurs at short distance with the substrate. Since a large part of this final energy gain is dissipated, this explains why more attractive substrate exhibit a larger $\hat{\mu}_{f}$.

It is interesting to contrast these results to our slip substrate. In a slip system the bottom layer is pulled along at similar speeds to the next layer. The dissipation will to a large extent stem from the liquid slipping across the substrate instead of from shear within the liquid wedge. The measurements shown in figure 4 are consistent with this picture.

\section{CONCLUSIONS}

We have investigated contact line advancement of a hydrophilic liquid, water, wetting a hydrophilic substrate. Due to the hydrogen bonding there is a no-slip boundary condition at the substrate interface. In a Navier-Stokes setting this is incompatible with a moving contact line. We have shown that the particle nature of the liquid resolves this incompatibility at the length scale of a single water molecule. Energy is dissipated in the processes that advance the contact line and we have shown that this accounts for a substantial part of the total energy dissipation in the viscous regime of wetting. The contact line moves forward through a combination of two different modes: (a) from a molecule rolling over the present contact line from an upper layer and (b) from one contact line molecule jumping into an adjacent lattice site of the substrate. This mode is MKT-like. The rolling mode dominates in the initial phase of wetting, whereas both modes occur with similar frequency in the viscous regime.

For the rolling mode, we have shown that molecules at the contact line form an obstacle, because they are hard and of fixed, finite size. Passing such obstacles requires a molecule, or group of molecules, to reach a sufficiently high thermal activation energy. Most of this energy is lost through dissipation after hydrogen bonding to the substrate. This results in additional energy dissipation at contact line. We relate the energy barrier to the dynamical geometry of the contact line and show that it yields good matches for the velocity in late-stage wetting (figure 7). Furthermore, we explain the difference in friction coefficient for differently charged substrates. This is because the acceleration of water molecules to the substrate is strongest at short distance, due to the $1 / r$ dependence of the electrostatic potential. After this final acceleration, water molecules are locked in place when hydrogen bonding with the substrate. Other water molecules cannot easily move around the locked molecules and a significant part of the gain in convective kinetic energy is then dissipated.

All effects described above arise because water molecules are largely immobilized when hydrogen bonding to a hydrophilic substrate. This is not the case for Lennard-Jones type systems that are commonly used to model wetting. In the latter case, liquid molecules can easily slip across the substrate which produces an entirely different mode of contact line advancement and much lower contact line friction. This is important to consider when modeling dynamic wetting at the molecular scale.

We are grateful to Andreas Carlson for discussions leading to this publication. This research was supported by the European Research Council (grant no. 258980) and the Swedish Research Council (grant no. 2014-4505). The simulations were performed on resources provided by the Swedish National Infrastructure for Computing (SNIC) at the PDC Center for High Performance Computing (SNIC 2015-10-30 and 2016-10-47). Analysis was performed and figures created using VMD [26] and MATPLOTLIB [27]. 


\section{APPENDIX: SIMULATION SET-UP}

Following our previous study [12, the simulation systems consist of a water droplet cylinder and an atomistic substrate. The no-slip substrate consists of rigid $\mathrm{SiO}_{2}$ tri-atomic "molecules" set in a hexagonal monolayer. For the substrate with slip, several layers of closely packed simple Lennard-Jones atoms are used. These simple atoms are non-charged and do not form hydrogen bonds with water molecules. See [12] for further details. The initial radius $R$ of the water droplets are $50 \mathrm{~nm}$ and their width $w=4.67 \mathrm{~nm}$. They consist of 1.2 million water molecules. The droplet and the substrate are equilibrated to a temperature $T=300 \mathrm{~K}$ using a stochastic dynamics integrator. After this stage a leap-frog MD integrator is used and wetting initiated by gently bringing the droplet within the interaction range of the substrate. The water droplet is not coupled to a thermostat during this stage of the simulation, but a velocity rescaling thermostat is applied to the substrate to dissipate heat with a time scale of $10 \mathrm{ps}$.

The water molecules are modeled using the $\mathrm{SPC} / \mathrm{E}$ model. At $300 \mathrm{~K}$ this has a viscosity of $8.77 \cdot 10^{-4}$ Pas, a density of $986 \mathrm{~kg} \mathrm{~m}^{-3}$ and a surface tension of $5.78 \cdot 10^{-2} \mathrm{~Pa} \mathrm{~m}$. Note that the surface tension is $20 \%$ slower than the experimental value. This is not an issue in this study, as we study different contact angles and they depend on relative differences between surfaces tensions. For the $\mathrm{SiO}_{2}$ atoms charges are varied to obtain different static contact angles $\theta_{0}$. We measured the contact angles by letting a small droplet relax on a substrate. The final equilibrium values are reported in table II. The $\mathrm{SiO}_{2}$ molecules are held in place by strong harmonic potentials on both oxygen atoms. Short range interactions are treated fully up to a cutoff of $0.9 \mathrm{~nm}$ and long range electrostatics using PME. Periodic boundary conditions are applied along the axes transverse to the substrate, along which the liquid spreads. Two repulsive potential walls are set along the remaining sides to stop molecules from escaping the system. A time step of $2 \mathrm{fs}$ is used for the integrator.

Simulations were run using Gromacs 2016 [28] in double precision on the Beskow supercomputer at KTH. When running on 1280 cores a speed of 5 ns/day was achieved.

* hess@kth.se

[1] P.-G. de Gennes, "Wetting: Statics and dynamics," Rev. Mod. Phys. 57, 827 (1985)

[2] D. Bonn, J. Eggers, J. Indekeu, J. Meunier, and E. Rolley, "Wetting and spreading," Rev. Mod. Phys. 81, 739805 (2009)

[3] J. H. Snoeijer and B. Andreotti, "Moving Contact Lines: Scales, Regimes, and Dynamical Transitions,"
Annu. Rev. Fluid Mech. 45, 269-292 (2013)

[4] A.-L. Biance, C. Clanet, and D. Quéré, "First steps in the spreading of a liquid droplet," Phys. Rev. E 69, 016301 (2004)

[5] J. C. Bird, S. Mandre, and H. A. Stone, "Short-Time Dynamics of Partial Wetting," Phys. Rev. Lett. 100, 234501 (2008)

[6] L. Courbin, J. C. Bird, M. Reyssat, and H. A. Stone, "Dynamics of wetting: from inertial spreading to viscous imbibition." J. Phys. Condens. Matter 21, 464127 (2009).

[7] F. Heslot, A. M. Cazabat, and P. Levinson, "Dynamics of wetting of tiny drops: Ellipsometric study of the late stages of spreading," Phys. Rev. Lett. 62, 1286-1289 (1989)

[8] L. Chen and E. Bonaccurso, "Effects of surface wettability and liquid viscosity on the dynamic wetting of individual drops," Phys. Rev. E 90, 022401 (2014).

[9] Y. Nakamura, A. Carlson, G. Amberg, and J. Shiomi, "Dynamic wetting at the nanoscale," Phys. Rev. E 88, 033010 (2013)

[10] A. Carlson, G. Bellani, and G. Amberg, "Universality in dynamic wetting dominated by contact-line friction," Phys. Rev. E 85, 045302 (2012).

[11] H. Perrin, R. Lhermerout, K. Davitt, E. Rolley, and B. Andreotti, "Defects at the Nanoscale Impact Contact Line Motion at all Scales," Phys. Rev. Lett. 116, 184502 (2016).

[12] P. Johansson, A. Carlson, and B. Hess, "Water-substrate physico-chemistry in wetting dynamics," J. Fluid Mech. 781, 695-711 (2015)

[13] M. J. de Ruijter, J. De Coninck, and G. Oshanin, "Droplet spreading: Partial wetting regime revisited," Langmuir 15, 2209-2216 (1999).

[14] A. Carlson, M. Do-Quang, and G. Amberg, "Dissipation in rapid dynamic wetting," J. Fluid Mech. 682, 213-240 (2011)

[15] J. De Coninck and T. D. Blake, "Wetting and Molecular Dynamics Simulations of Simple Liquids," Annu. Rev. Mater. Res. 38, 1-22 (2008)

[16] T. A. Ho, D. V. Papavassiliou, L. L. Lee, and A. Striolo, "Liquid water can slip on a hydrophilic surface," Proc. Natl. Acad. Sci. USA 108, 16170-16175 (2011)

[17] K. G. Winkels, J. H. Weijs, A. Eddi, and J. H. Snoeijer, "Initial spreading of low-viscosity drops on partially wetting surfaces," Phys. Rev. E 85, 055301 (2012).

[18] J. E. Andrews, S. Sinha, P. W. Chung, and S. Das, "Wetting dynamics of a water nanodrop on graphene," Phys. Chem. Chem. Phys. 18, 23482-23493 (2016).

[19] S. Liu, X. Yang, and Y. Qin, "Molecular dynamics simulation of wetting behavior at $\mathrm{CO} 2 /$ water/solid interfaces," Chin. Sci. Bull. 55, 2252-2257 (2010).

[20] Q. Yuan and Y.-P. Zhao, "Precursor film in dynamic wetting, electrowetting, and electro-elasto-capillarity," Phys. Rev. Lett. 104, 246101 (2010).

[21] K. Ritos, N. Dongari, M. K. Borg, Y. Zhang, and J. M. Reese, "Dynamics of nanoscale droplets on moving surfaces." Langmuir 29, 6936-43 (2013).

[22] H. J. C. Berendsen, J. R. Grigera, and T. P. Straatsma, "The missing term in effective pair potentials," J. Phys. Chem. 91, 6269-6271 (1987)

[23] A. Carlson, G. Bellani, and G. Amberg, "Contact line dissipation in short-time dynamic wetting," Europhys. Lett. 97, 44004 (2012)

[24] P. Yue and J. J. Feng, "Wall energy relaxation in the 
Cahn-Hilliard model for moving contact lines," Phys. Fluids 23, 012106 (2011)

[25] T. D. Blake and J. M. Haynes, "Kinetics of Liquid/Liquid Displacement," J. Colloid Interface Sci. 80, 1-3 (1969)

[26] W. Humphrey, A. Dalke, and K. Schulten, "VMD - Visual Molecular Dynamics," J. Mol. Graphics 14, 33-38 (1996)
[27] J. D. Hunter, "Matplotlib: A 2d graphics environment," Computing in Sci. \& Eng. 9, 90-95 (2007).

[28] M. J. Abraham, T. Murtola, R. Schulz, S. Páll, J. C. Smith, B. Hess, and E. Lindahl, "Gromacs: High performance molecular simulations through multi-level parallelism from laptops to supercomputers," SoftwareX 1-2, 19-25 (2015) 